\title{
Morphic Angles
}

\section{Mike Vanderroost ${ }^{1}$}

\begin{abstract}
The golden ratio and the plastic number are both so-called morphic numbers that have been studied in the past in various scientific domains, in particular in architecture. Based on the golden ratio, the concept of a golden angle has been defined for a circle in two-dimensional Euclidean space. However, at present, there exists no three-dimensional analog based on the plastic number. In this paper, the concept of morphic angles will be introduced, based on morphic numbers. New definitions will be proposed for these angles that are applicable for nondegenerated quadratic curves and surfaces of revolution, respectively.
\end{abstract}

Keywords Golden ratio $\cdot$ Plastic number $\cdot$ Golden morphic angle $\cdot$ Plastic angle $\cdot$ Plastic morphic angle

\section{Introduction}

The golden ratio $\varphi=\frac{1+\sqrt{5}}{2} \approx 1.618$ and the plastic (or van der Laan's) number $\psi \approx 1.325^{1}$ are the only two so-called morphic numbers $p \in \mathbb{R}$ greater than 1 that satisfy the following equations (Aarts et al. 2001):

$$
p+1=p^{k}
$$

1 The exact value of the plastic number is $\frac{\sqrt[3]{108+12 \sqrt{69}}+\sqrt[3]{108+12 \sqrt{69}}}{6}$.

Mike Vanderroost

mike.vanderroost.work@gmail.com

1 Ghent, Belgium 


$$
p-1=p^{-l}
$$

with $k$ and $l \in \mathbb{N}$. While the golden ratio is already known to mankind since antiquity and still is a subject of study in art, architecture (Xu et al. 2012; Huylebrouck and Labarque 2002) and various scientific domains such as mathematics, physics, biology and chemistry (Gonzlez 2010; Tung 2007; Yu et al. 2006; Swartzendruber et al. 1985; Boeyens and Thackeray 2014), the plastic number $\psi$ was first defined and studied mathematically in 1924 by the engineer Gerard Codonnier. The first publication about this number, however, dates from 1960. That year, Dom Hans van der Laan $^{2}$ presented the plastic number through a new measurement scale of architectural proportions that is solely based on an empirical relation between the plastic number and the human capacity to visually perceive, distinguish and relate dimensions in threedimensional architectural space (Padovan 2002). As opposed to Le Corbusier's well known Modulor, van der Laan's measurement scale of architectural proportions does not take into account human dimensions, nor does it focus on the convenience of architectural spaces.

The main goal of this paper is to introduce the concept of so-called morphic angles, i.e. angles defined based on the morphic numbers. First, it will be explained how, based on the golden ratio, a morphic angle can be defined for all types of bivariate nondegenerated quadratic curves in two-dimensional Euclidean space. Subsequently, the concept of a morphic angle based on the plastic number will be introduced, defined and extensively discussed. Finally, it will be shown that through the concept of a morphic angle, a formula can be derived based on the plastic number that approaches the number $\pi$ with good accuracy.

\section{The Circle-Based Definition of a Golden Angle}

From a geometrical point of view, the golden ratio emerges from the following relation between the dimensions of a so-called golden rectangle in two-dimensional Euclidean space:

$$
\varphi=\frac{b}{a}=\frac{a+b}{b}
$$

with $a$ and $b$ the length and the width of the golden rectangle, respectively. From the above relation, it can be derived that the golden ratio satisfies the following equations:

$$
\begin{gathered}
\varphi+1=\varphi^{2} \\
\varphi-1=\frac{1}{\varphi}
\end{gathered}
$$

Now, consider the total circumference $C_{c}$ of a circle with radius $r$ that is sectioned according to the golden ratio (in literature this is often denoted as a golden section).

\footnotetext{
${ }^{2}$ Architect and member of the Benedictine order.
} 
Fig. 1 A golden angle defined on a circle

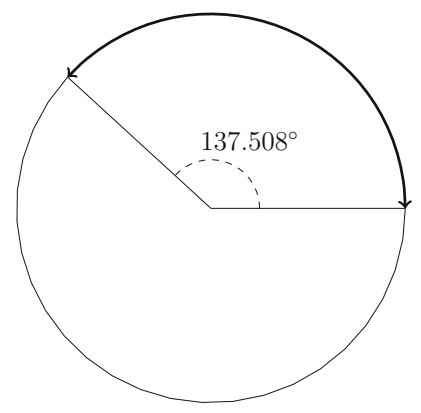

This results in a so-called golden arc with length $C_{\varphi}$ and an arc with length $C^{\prime}=$ $\varphi C_{\varphi}$ (see Fig. 1). Using Eq. (4), it can be determined that

$$
C_{\varphi}=\frac{C_{c}}{\varphi^{2}}=\frac{2 \pi r}{\varphi^{2}} \approx 2.400 r
$$

A golden angle has been defined in two-dimensional Euclidean space as the central angle subtended by a golden arc and has a value of approximately $137.508^{\circ}$ (Prusinkiewicz and Lindenmayer 1990). To date, a golden angle has been studied in various scientific domains such as physics (Livio 2003) and medicine (Henein et al. 2011).

\section{Towards a Definition of a Golden Morphic Angle}

\section{Introduction}

A circle can be regarded as the simplest type of a conic section, i.e. a bivariate nondegenerated quadratic curve (BNQC) in two-dimensional Euclidean space. It is therefore worthwhile investigating whether or not a golden morphic angle can be defined that is based on the golden ratio and is applicable for any BNQC type, more specifically a circle, ellipse, hyperbola, and parabola. Therefore, consider the general polar equation of a BNQC with origin at a focus point $P_{i}$ of a BNQC: ${ }^{3}$

$$
r=\frac{e p}{1+e \cos v_{i}}
$$

with $e$ the eccentricity and $p$ the focal parameter of a BNQC (see Table 1). The focal radius with length $r$ is the line segment between the considered focus point and a point on a BNQC. The true anomaly $v_{i}$ at focus point $P_{i}$ of a point $P$ on a BNQC is the angle measured between its focal radius and the minimal focal radius with length $r_{\min }$ corresponding to a point $P_{\min }$ on the BNQC closest to the focus point (see Fig. 2).

For a circle, both focus points coincide with the center of the circle, implying that the central angle in the circle-based definition of a golden angle can be

\footnotetext{
$\overline{3}$ For an ellipse, a circle and a hyperbola $i \in\{1,2\}$, for a parabola $i=1$.
} 
Table 1 Eccentricity $e$, focal parameter $p$ and minimum focal radius $r_{\min }$ for each BNQC type

\begin{tabular}{llll}
\hline BNQC type & Eccentricity $e$ & Focal parameter $p$ & $r_{\min }$ \\
\hline Circle & 0 & $\infty$ & $a$ \\
Ellipse & ] $0,1[$ & $a\left(1-e^{2}\right) / e$ & $a(1-e)$ \\
Parabola & 1 & $2 a / e$ & $a$ \\
Hyperbola & ] $1, \infty[$ & $a\left(e^{2}-1\right) / e$ & $a(1+e)$
\end{tabular}

Fig. 2 Characteristics of a BNQC

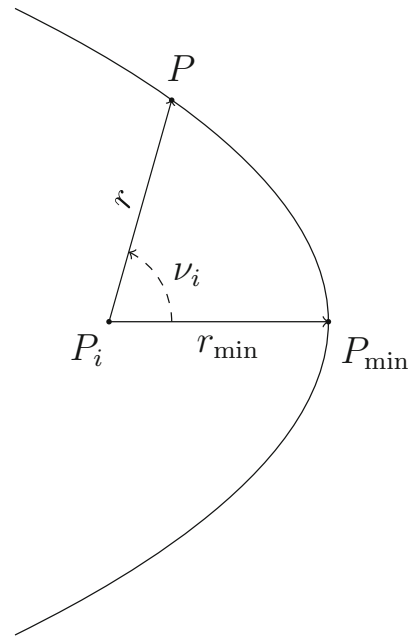

regarded as the difference $\Delta v$ between the true anomalies corresponding with the end and starting point of a golden arc, respectively. Therefore, $\Delta v$ at a certain focus point of a BNQC will be considered in the search for a definition of a golden morphic angle.

\section{Limitations of the Circle-Based Definition}

If a golden morphic angle of a BNQC would be defined as the $\Delta v$ related to a golden arc resulting from a golden section of the total circumference of a BNQC, three major problems would arise. Firstly, hyperbolas and parabolas are non-closed BNQCs, meaning that they do not have a finite circumference. Secondly, for ellipses, hyperbolas and parabolas, there is no exact formula to calculate the length of an arc segment, implying that the value of the corresponding $\Delta v$ cannot be exactly determined. Finally, the value of $\Delta v$ depends on the location of an arc segment with fixed length along the BNQC, implying that $\Delta v$ does not have a unique value. It is thus clear that the circle-based definition of a golden angle cannot be applied for all BNQC types to define a golden morphic angle. 


\section{Proposal of a New Definition}

In the circle-based definition, a golden angle is defined based on a ratio of two lengths equal to the golden ratio. A similar approach will be pursued in the search for a definition of a golden morphic angle. For that reason, consider two crosssections perpendicular to the minimal focal radius at a focus point $P_{i}$ of a BNQC (see Fig. 3). Each cross-section intersects the BNQC in two points. For the first cross-section, the perpendicular distance between the points of intersection $P_{1}^{\prime}$ and $P_{2}^{\prime}$ on the BNQC and the minimal focal radius is equal to the focal radius $r^{\prime}$ for which $v_{i}=90^{\circ}$. The second cross-section (from now on denoted as the golden cross section) is chosen such that the perpendicular distance $r_{\varphi}$ between the points of intersection $P_{1}^{\varphi}$ and $P_{2}^{\varphi}$ on the BNQC and the minimum focal radius relates to $r^{\prime}$ as

$$
\frac{r^{\prime}}{r_{\varphi}}=\varphi
$$

The above expression clearly respresents a golden ratio of two lengths. A golden morphic angle $\theta_{\varphi}$ at a focus point $P_{i}$ of a BNQC can now be defined as the apex angle $2 v_{\varphi}<180^{\circ}$ of the isosceles triangle formed by the focus point and the points of intersection resulting from the golden cross-section (see Fig. 3). From Eqs. (7) and (8) it can be derived that

$$
r_{\varphi}=\frac{e p}{\varphi}=\frac{e p \sin v_{\varphi}}{1+e \cos v_{\varphi}}
$$

This results in the following quadratic equation for $v_{\varphi}$

$$
\left(e^{2}+\varphi^{2}\right) \cos ^{2} v_{\varphi}+2 e \cos v_{\varphi}+1-\varphi^{2}=0
$$

Since by definition $v_{\varphi}<90^{\circ}$, only the root resulting in a positive value of $\cos v_{\varphi}$ is considered. It can be derived that the value of $\theta_{\varphi}$ is determined through the following formula:

Fig. 3 A golden morphic angle defined on a BNQC

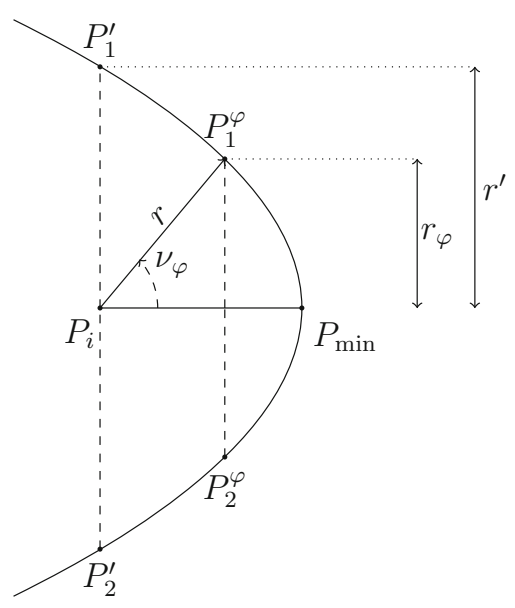


Table 2 Approximate (range of) values of a golden morphic angle $\theta_{\varphi}$ for each BNQC type

\begin{tabular}{ll}
\hline BNQC type & Golden morphic angle $\theta_{\varphi}$ \\
\hline Circle & $\approx 76.346^{\circ}$ \\
Ellipse & $>76.346^{\circ}$ and $<126.871^{\circ}$ \\
Parabola & $\approx 126.871^{\circ}$ \\
Hyperbola & $>126.871^{\circ}$ and $<180^{\circ}$ \\
\hline
\end{tabular}

$$
\theta_{\varphi}=2 \arccos \left(\frac{-e+\varphi \sqrt{\varphi+e^{2}}}{e^{2}+\varphi^{2}}\right)
$$

With the proposed definition, a golden morphic angle is exactly and uniquely defined for all BNQC types. The approximate (range of) value(s) of a golden morphic angle for each BNQC type are listed in Table 2.

For completeness, it is important to mention here that the cancellation of the factors ep in Eq. (9) is not allowed for a circle because $e=0$ and $p=\infty$, implying that Eq. (11) cannot be mathematically derived for this particular BNQC. However, it can be easily verified that the value of the golden morphic angle obtained by applying basic goniometry $\left(r^{\prime}=r\right.$, implying that $\left.\sin v_{\varphi}=\frac{1}{\varphi}\right)$ is equal to the value obtained through Eq. (11) for $e=0$. Moreover, it can be noticed that the morphic golden angle for a circle is part of a class of angles related to the concept of a golden triangle (i.e., an isosceles triangle with sides $\varphi, \varphi$, and 1). In this particular case, the angle is related to a so-called golden morphic triangle with sides $\varphi, \varphi$, and 2 .

\section{A Sphere-Based Definition of a Plastic Angle}

The plastic number $\psi$ is the real solution of the equation $x^{3}-x-1=0$. It can be considered as the golden number's analogon in three-dimensional Euclidean space. It emerges from the following relation between the dimensions of a so-called plastic rectangular cuboid (also denoted with the terms plastic box or $\psi$-box (de Spinadel and Buitrago 2009):

$$
\psi=\frac{b}{a}=\frac{c}{b}=\frac{a+b}{c}
$$

with $a, b$, and $c$ the length, the width, and the height of the rectangular cuboid, respectively. From the above relation, it can be derived that the plastic number satisfies the following equation:

$$
\psi+1=\psi^{3}
$$

From the equality $x^{5}-x^{4}-1=\left(x^{3}-x-1\right)\left(x^{2}-x+1\right)$ it follows that $\psi$ is also a solution of the equation $x^{5}-x^{4}-1=0$, implying that: 


$$
\psi-1=\frac{1}{\psi^{4}}
$$

The plastic number is thus a morphic number.

Analogously to the circle-based definition of a golden angle, it is quite straightforward to define a plastic angle in three-dimensional Euclidean space. Consider a sphere with radius $R$ and section its total area $A_{s}$ in two areas $A_{\psi}$ and $A^{\prime}$ [see Eq. (4)] such that

$$
\frac{A^{\prime}}{A_{\psi}}=\psi
$$

Using Eq. (13), it can be determined that

$$
A_{\psi}=\frac{A_{s}}{\psi^{3}}=\frac{4 \pi R^{2}}{\psi^{3}} \approx 5.406 R^{2}
$$

A spherical surface having an area equal to $A_{\psi}$ will from now on be referred to as a plastic surface $S_{\psi}$. Since any solid angle $\Omega$ at the center of a sphere is related to the area $A_{\Omega}$ of a surface on a sphere that subtends it $\left(A_{\Omega}=\Omega R^{2}\right)$, a plastic angle $\Omega_{\psi}$ can now be defined as the solid angle subtended at the center of a sphere by the area of a plastic surface $S_{\psi}$ (see Fig. 4). The proposed sphere-based definition of a plastic angle $\Omega_{\psi}$ can thus be considered as the three-dimensional analogon of the circlebased definition of a golden angle. In spherical coordinates, this can be written as:

$$
\Omega_{\psi}=\iint_{S_{\psi}} \sin \theta \mathrm{d} \theta \mathrm{d} \phi \approx 5.406
$$

with $\theta \in[0, \pi]$ the colatitude and $\phi \in[0,2 \pi]$ the longitude. The previous equation can be rewritten as

$$
\Omega_{\psi}=\int_{\theta_{1}}^{\theta_{2}} \sin \theta \mathrm{d} \theta \int_{\phi_{1}}^{\phi_{2}} \mathrm{~d} \phi=\left(\phi_{2}-\phi_{1}\right)\left(\cos \theta_{1}-\cos \theta_{2}\right)
$$

with $\left(\theta_{1}, \theta_{2}\right)$ and $\left(\phi_{1}, \phi_{2}\right)$ the boundary values of $\theta$ and $\phi$, respectively. For a plastic angle, these values need to satisfy the following conditions:

$$
\begin{gathered}
\phi_{2}-\phi_{1} \in\left[\frac{2 \pi}{\psi^{3}}, 2 \pi\right] \\
\cos \theta_{1}-\cos \theta_{2} \in\left[\frac{2}{\psi^{3}}, 2\right]
\end{gathered}
$$

Because each set of boundary values satisfying the above conditions corresponds to a plastic angle with different shape and/or location, $\Omega_{\psi}$ is not uniquely defined in terms of the latter properties. Assuming that angle location is a relative property (just like in the circle-based definition of a golden angle), $\Omega_{\psi}$ can be uniquely defined by constraining the shape of a plastic surface to a specific spherical surface. In the appendix, plastic surfaces and related volumes are discussed based on the 
Fig. 4 A plastic angle defined on a sphere

following spherical surfaces: zone, cap, lune, and polygon. Because the considered spherical surfaces are extensively described in various handbooks of spherical geometry (Bronshtein et al. 2004), they will only be briefly introduced.

\section{Towards a Definition of a Plastic Morphic Angle}

\section{Introduction}

A sphere can be considered as the simplest type of a nondegenerated real quadratic surface in three-dimensional Euclidean space that results from revolving a BNQC around an axis of symmetry going through the focus point(s). These surfaces will from now on be denoted as quadratic surfaces of revolution (QSR). There are four types of such surfaces: spheres, spheroids, paraboloids, and two-sheet hyperboloids. ${ }^{4}$ For each QSR, the cross-section perpendicular to the axis of symmetry results in a circle. Similar to the circle-based definition of golden angle, the solid angle considered in the sphere-based definition of a plastic angle can be regarded as the solid angle at the coinciding focus points of the revolved circle. Therefore, a solid angle at a focus point of the revolved BNQC will be considered in the search for a definition of a plastic morphic angle of a QSR.

\section{Limitations of the Proposed Sphere-Based Definition}

If the proposed sphere-based definition of a plastic angle would be applied for each QSR type, a similar problem would arise as in the case of a golden angle (see Sect. 3.2): for paraboloids and two-sheet hyperboloids, the total area of the surface is infinite, meaning that it is impossible to realize a section based on the plastic number. It is thus clear that the sphere-based definition is not generally applicable.

\footnotetext{
${ }^{4}$ One-sheet hyperboloids will not be considered in this section, because their axis of symmetry is perpendicular to the axis of symmetry going through the focus points.
} 


\section{Proposal of a New Definition}

In the proposed sphere-based definition, a plastic angle is defined based on a ratio of two areas equal to the plastic number [see Eq. (15)]. A similar approach will be pursued in the search for a definition of a plastic morphic angle. Therefore, consider two cross-sections of a QSR perpendicular to the axis of symmetry (see Fig. 5). The first cross-section goes through a focus point $P_{i}$. The radius $r^{\prime}$ of the resulting circle $C^{\prime}$ is equal to the focal radius in Eq. (7) with $v_{i}=90^{\circ}$. The second cross-section is chosen such that the radius $r_{\psi}$ of the resulting circle $C_{\psi}$ (from now on referred to as a plastic circle) relates to $r^{\prime}$ as

$$
\left(\frac{r^{\prime}}{r_{\psi}}\right)^{2}=\psi
$$

The above expression respresents a ratio of two areas of surfaces equal to the plastic number. A plastic morphic angle $\Omega_{\psi}$ of a QSR is now defined as the solid angle at a focus point of the revolved BNQC subtended by a plastic circle. This corresponds to the solid angle subtended by a cone with apex angle $2 v_{\psi}<180^{\circ}$ (see Fig. 5). From Eqs. (21) and (7) it can be derived that

$$
r_{\psi}=\frac{e p}{\sqrt{\psi}}=\frac{e p \sin v_{\psi}}{1+e \cos v_{\psi}}
$$

This results in the following quadratic equation for $v_{\psi}$

$$
\left(e^{2}+\psi\right) \cos v_{\psi}^{2}+2 e \cos v_{\psi}+1-\psi=0
$$

Since by definition $v_{\psi}<90^{\circ}$, only the root resulting in a positive value of $\cos v_{\psi}$ is considered. It can be derived that the value of $v_{\psi}$ is determined through the following formula:

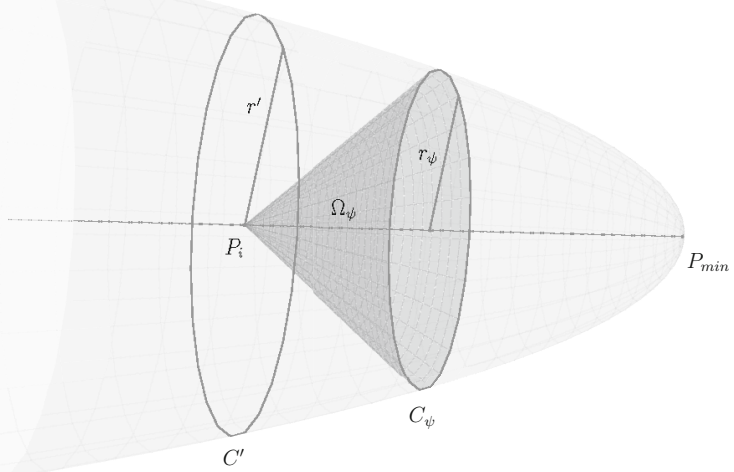

Fig. 5 A plastic angle defined on a QSR 
Table 3 Approximate (range of) values of $v_{\psi}$ and $\Omega_{\psi}$ for each QSR type

\begin{tabular}{lll}
\hline QSR type & $v_{\psi}$ & $\Omega_{\psi}$ \\
\hline Sphere & $\approx 60.344^{\circ}$ & $\approx 3.174$ \\
Spheroid & $>60.344^{\circ}$ and $<81.971^{\circ}$ & $>3.174$ and $<5.406$ \\
Paraboloid & $\approx 81.971^{\circ}$ & $\approx 5.406$ \\
2-Sheet hyperboloid & $>81.971^{\circ}$ and $<90^{\circ}$ & $>5.406$ and $<2 \pi$ \\
\hline
\end{tabular}

$$
v_{\psi}=\arccos \left(\frac{-e+\sqrt{\psi\left(\psi+e^{2}-1\right)}}{e^{2}+\psi}\right)
$$

The plastic morphic angle of a QSR can then be calculated through the following formula for the solid angle subtended by a cone;

$$
\Omega_{\psi}=2 \pi\left(1-\cos v_{\psi}\right)
$$

The approximate (range of) values of $v_{\psi}$ and $\Omega_{\psi}$ are listed in Table 3 for all QSR types. It is immediately apparent that the plastic morphic angle for a paraboloid obtained with the general definition is equal to the plastic angle obtained through the sphere-based definition. Analog to the golden morphic angle, the cancellation of the factors $e p$ in Eq. (22) is not allowed for a circle, implying that Eq. (24) cannot be mathematically derived for the related revolved BNQC. Again, it can be easily verified that the value of $v_{\psi}$ obtained by applying basic goniometry $\left(r^{\prime}=r\right.$, implying that $\sin v_{\psi}=\frac{1}{\sqrt{\psi}}$ ) is equal to the value obtained through Eq. (24) for $e=0$.

\section{A Plastic Approximation of $\pi$}

The total area $A_{\psi}^{b o x}$ of the surface of a plastic box equals

$$
A_{\psi}^{\text {box }}=2\left(\psi^{3}+\psi^{2}+\psi\right) a^{2} \approx 10.808 a^{2}
$$

with $a$ being the length of the smallest dimension of the plastic box. When comparing this with the area $A_{\psi}$ of a plastic surface on a sphere with radius $R$ [see Eq. (16)], it can be seen that if $a=R=1$, it holds that

$$
\frac{A_{\psi}^{b o x}}{A_{\psi}}=\frac{\psi^{6}+\psi^{5}+\psi^{4}}{2 \pi} \approx 2
$$

with an accuracy of $2.10^{-6}$. This means that $\pi$ can be approximated with the same accuracy by a sum of powers of $\psi$ as follows:

$$
\pi \approx \pi_{\psi}=\frac{\psi^{6}+\psi^{5}+\psi^{4}}{4}=3.140876 \ldots
$$

with $\pi_{\psi}$ the plastic approximation of $\pi$. Using Eq. (13), $\pi_{\psi}$ can be rewritten as 


$$
\pi \approx \pi_{\psi}=\frac{\psi^{9}}{4}
$$

The difference between the real value of $\pi$ and $\pi_{\psi}$ amounts to $0.0007166 \ldots$. When comparing this difference with the area $A^{\prime}=A_{s}-A_{\psi}=4 \pi / \psi^{2}$ of the surface considered in Sect. 4 , it can be noticed that $\pi-\pi_{\psi}$ is approximately $10^{-4} A^{\prime}$. The plastic approximation of $\pi$ can thus be significantly improved by taking into account the latter in Eq. (29), resulting in

$$
\pi \approx \pi_{\psi}=\frac{7 \psi^{2}+9 \psi+5}{4+3.9984 \psi}=3.141592 \ldots
$$

This improved plastic approximation of $\pi$ has an accuracy of $1.10^{-9}$. The difference between the real value of $\pi$ and $\pi_{\psi}$ is now reduced to $0.0000003643 \ldots$.

\section{Conclusion}

In this paper, the concept of morphic angles was introduced. Morphic angles were defined based on the morphic numbers for nondegenerated quadratic curves and surfaces of revolution. Notwithstanding that these angles were presented as new theoretical concepts, the author of this paper considers it possible that they have a deeper meaning in various scientific domains, especially in physics were the considered curves and surfaces are often encountered (particle motion, force fields, astrophysics, ...). Moreover, the author believes that the morphic angles defined in this paper may be of practical use in architectural design and construction.

Acknowledgments The author would like to thank professor Bernard De Baets (Research Unit Knowledge-based Systems, Ghent University, Belgium) and Thomas Van der Velde (School of Arts, Belgium) for their time and feedback. Any findings and conclusions are those of the author and do not necessarily reflect those of the above-mentioned persons.

\section{Appendix 1: Plastic Surfaces with a Specific Shape}

\section{Plastic Zone and Plastic Cap}

A plastic zone (see Fig. 6a) is a plastic surface comprised between two parallel planes cutting a sphere. It can determined that the height $h_{z, \psi}$ of a plastic zone equals $2 R / \psi^{3}$, implying that the boundaries in Eq. (18) satisfy the following relations

$$
\begin{gathered}
\phi_{2}-\phi_{1}=2 \pi \\
\cos \theta_{1}-\cos \theta_{2}=\frac{2}{\psi^{3}}
\end{gathered}
$$

If one of the cutting planes is tangent to the surface of the sphere, the resulting plastic zone is a plastic cap (see Fig. 6b). The area $A_{b a s e, \psi}$ of the base circle of a plastic cap relates to $A_{\psi}$ and $A_{s}$ as follows: 


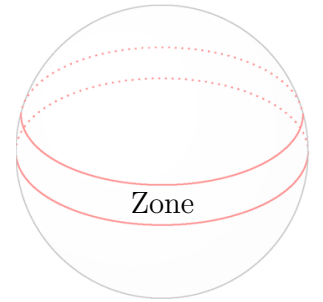

(a)

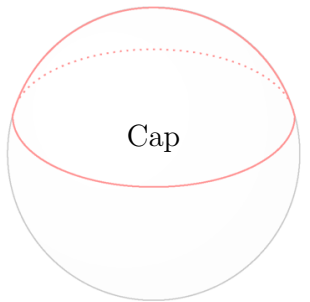

(b)

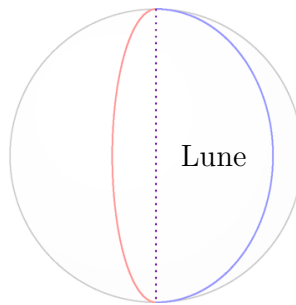

(c)

Fig. 6 Plastic surfaces

$$
A_{\psi}^{\text {base }}=\frac{A_{\psi}}{\psi^{2}}=\frac{A_{s}}{\psi^{5}}
$$

The volume enclosed by a plastic cap and its base circle relates to a sphere's volume as

$$
V_{\psi}^{c a p}=\frac{V_{s}(3 \psi+1)}{\psi^{9}}
$$

\section{Plastic Cone and Plastic Sector}

A plastic cone is a circular cone with plastic apex angle $\delta_{\psi}$ at the center of a sphere and a base circle equal to that of a plastic cap. It can be derived that $\delta_{\psi}$ equals

$$
\delta_{\psi}=\arccos \left(\frac{1}{\psi^{7}}\right) \approx 81.971^{\circ}
$$

A plastic sector is a spherical sector obtained by composing a plastic cap and its corresponding plastic cone. This is an example of a spherical volume element constructed by connecting the contour of a certain spherical surface with the sphere's center. The volume $V_{\Omega}$ of such an element can be directly calculated from the solid angle $\Omega$ subtended by the spherical surface:

$$
V_{\Omega}=\frac{\Omega R^{3}}{3}=\frac{A_{\Omega} R}{3}
$$

This implies that the volume $V_{\psi}^{\text {sector }}$ of a plastic sector relates to a sphere's volume as

$$
V_{\psi}^{\text {sector }}=\frac{V_{s}}{\psi^{3}}
$$

The relation described in Eq. (16) for the area of a plastic surface thus also holds for the volume of a plastic sector, and by extension all the plastic volumes that will be discussed hereafter. For completeness, the area of the surface of a plastic sector is given: 


$$
A_{\psi}^{\text {sector }}=A_{\psi}+\pi R \sqrt{\frac{A_{\psi}^{\text {base }}}{\pi}}=\pi R^{2} \frac{(4+2 \sqrt{\psi})}{\psi^{3}}
$$

\section{Plastic Lune and Plastic Wedge}

A plastic lune is a plastic surface comprised between two planes cutting a sphere and intersecting each other in a line through the sphere's center (see Fig. 6c) and rotated relative to each other by a dihedral angle $\left.{ }^{5} \psi_{\psi} \in\right] 0,2 \pi[$. It can be determined that the plastic dihedral angle $\psi_{\psi}$ of plastic lune equals approximately $2 \pi /(1+\psi)$ $\operatorname{rad}$ or $154,857^{\circ}$. This means that the boundaries in Eq. (18) satisfy the following relations:

$$
\begin{gathered}
\phi_{2}-\phi_{1}=\frac{2 \pi}{\psi^{3}} \\
\theta_{2}-\theta_{1}=\pi
\end{gathered}
$$

A plastic wedge is the volume enclosed by two great ${ }^{6}$ semicircles and a plastic lune. The area of its surface equals

$$
A_{\psi}^{\text {wedge }}=A_{\psi}+\pi R^{2}
$$

\section{Plastic Polygon}

A plastic polygon is a plastic surface comprised between $n$ planes cutting a sphere and going through the sphere's center. Its so-called spherical angles correspond with the dihedral angles between the cutting planes. To verify whether or not it is possible to construct a plastic polygon, the general formula for the area $A_{\text {poly }}$ of a spherical polygon can be used (Calladine 1989):

$$
A_{\text {poly }}=(\sigma-(n-2) \pi) R^{2}
$$

with $\sigma \in](n-2) \pi, n \pi\left[\right.$ the sum of all radian spherical angles $\left.\psi_{i} \in\right] 0, \pi[$ with $i \in[1, n]$. From this formula, it can be derived that the sum of the radian spherical angles $\sigma_{\psi}$ in a plastic polygon equals

$$
\sigma_{\psi}=n \pi-\frac{2 \pi}{\psi^{7}}<n \pi \quad \text { for any } n \in \mathbb{N}
$$

implying that a plastic polygon can always be constructed. From Eq. (43) it can be derived that for any regular plastic polygon ${ }^{7}$ the magnitude of each spherical angle $\psi_{\psi}^{r e g}$ is

\footnotetext{
5 A dihedral angle is an angle created by two intersecting planes.

6 A great circle is the intersection of a sphere and a plane going through the sphere's center.

7 A regular plastic polygon has $n$ sides of equal length and $n$ spherical angles of equal magnitude.
} 


$$
\psi_{\psi}^{r e g}=\pi-\frac{2 \pi}{n \psi^{7}}
$$

If $n \rightarrow \infty$, the magnitude of $\psi_{\psi}^{r e g}$ will approach $\pi$. The length $a_{\psi}^{\text {reg }}$ of the sides of a regular plastic polygon can be determined through the following equation (Rajpoot 2015):

$$
a_{\psi}^{r e g}=2 R \arccos \left(\frac{\cos \frac{\pi}{n}}{\cos \frac{\psi_{\psi}^{r e g}}{2}}\right)
$$

In the specific case of a plastic triangle, it is important to mention here that some plastic triangles cannot be constructed. For example, right-angled plastic triangles (i.e., plastic triangles with one, two or three right spherical angles) cannot be constructed because they do not meet the conditions for the spherical angles. Oblique plastic triangles ${ }^{8}$ can only be constructed if the smallest spherical angle is greater than approximately $0.721 \pi \mathrm{rad}$ or $129,715^{\circ}$. Such oblique plastic triangles can be equilateral (or regular), isosceles or scalene.

\section{Plastic Pyramid}

A plastic pyramid is as a pyramid with apex at the center of a sphere and a plastic polygon as a base. It can be derived that the total area $A_{\psi}^{\text {pyramid }}$ of a plastic pyramid equals

$$
A_{\psi}^{\text {pyramid }}=A_{\psi}+\sum_{i=1}^{n} A_{i}^{\text {side }}=A_{\psi}+\frac{R}{2} \sum_{i=1}^{n} a_{i}
$$

with $A_{i}^{\text {side }}=R a_{i} / 2$ the area of a circular sector related to the length $a_{i}$ of a side of the plastic polygon.

\section{References}

Aarts, J., Fokking, R., and Kruijtzer, G. (2001). Morphic numbers. pages 56-58.

Boeyens, J. C. and Thackeray, J. F. (2014). Number theory and the unity of science. 110:1-2.

Bronshtein, I., Semendyayev, K., Musiol, G., and M++hlig, H. (2004). Handbook of Mathematics. Springer; 4th edition.

Calladine, C. R. (1989). Theory of Shell Structures. Cambridge University Press.

de Spinadel, V. W. and Buitrago, A. R. (2009). Towards van der laans plastic number in the plane. 13:163-175.

Gonzlez, A. (2010). Measurement of areas on a sphere using fibonacci and latitude-longitude lattices. 42:49-64.

Henein, M. Y., Zhao, Y., Nicoll, R., Sun, L., Khir, A. W., Franklin, K., and Lindqvist, P. (2011). The human heart: Application of the golden ratio and angle. 150:239-242.

\footnotetext{
$\overline{8}$ An oblique plastic triangle is a plastic triangle without right angles.
} 
Huylebrouck, D. and Labarque, P. (2002). More true applications of the golden number. 4:45-58.

Livio, M. (2003). The Golden Ratio: The Story of PHI, the World's Most Astonishing Number. Broadway Books.

Padovan, R. (2002). Dom Hans Van Der Laan and the Plastic Number, pages 181-193. Kim Williams Books.

Prusinkiewicz, P. and Lindenmayer, A. (1990). The algorithmic beauty of plants. Springer-Verlag.

Rajpoot, H. C. (2015). Mathematical analysis of regular spherical polygons by using hcr's theory of polygon (spherical geometry by h.c. rajpoot).

Swartzendruber, L., Schectman, D., Bendersky, L., and Cahn, J. W. (1985). Nuclear gamma-ray resonance observations in an aluminum-based icosahedral quasicrystal. 32:1383-1385.

Tung, K. K. (2007). Topics in Mathematical Modeling. Princeton University Press.

$\mathrm{Xu}$, L., Yang, C. G., and Wei, T. C. (2012). Application of the golden section and golden rectangle to fountain location in landscape design. 174-177:2554-2557.

Yu, D., Xue, D., and Ratajczak, H. (2006). Golden ratio and bond-valence parameters of hydrogen bonds of hydrated borates. 783:210-214.

Mike Vanderroost holds a degree in Engineering Physics and a Ph.D. in Bioscience Engineering. His work covers many fields, ranging from quantum physics and reactor modelling to intelligent packaging and software systems. Througout his education and career, he has always been interested in architecture and its link with mathematics. 